\title{
Ultra-broadband mobile networks from LTE-Advanced to 5G: evaluation of massive MIMO and multi-carrier aggregation effectiveness
}

\author{
Marco Neri, Maria-Gabriella Di Benedetto \\ Dept. of Information Engineering, \\ Electronics and Telecommunications (DIET) \\ Sapienza, Università di Roma \\ Roma, Italia \\ neri.1480553@studenti.uniroma1.it \\ mariagabriella.dibenedetto@uniroma1.it
}

\author{
Tommaso Pecorella \\ Dept. of Information Engineering \\ Università di Firenze \\ Firenze, Italia \\ tommaso.pecorella@unifi.it
}

\author{
Camillo Carlini, Andrea Castellani, \\ Pietro Obino, Pamela Sciarratta \\ Telecom Italia S.p.A. \\ Roma, Italia \\ camillo.carlini@telecomitalia.it \\ andrea.castellani@telecomitalia.it \\ pietro.obino@telecomitalia.it \\ pamela.sciarrattaarmanicampanella@telecomitalia.it
}

\begin{abstract}
LTE-Advanced networks are spreading widely across the world and they are continuing to evolve as new device features such MIMO 4x4, Carrier Aggregation are being released to move towards the peak data rates introduced by 3GPP Release 12 and 13. Mobile network Operators are looking for technologies that guarantee higher spectral efficiency and wider spectrum usage but they have to deal with limitations due to commercial devices' RF components. This paper analyzes several scenarios, compares them and suggests deployment strategies.

Index Terms-LTE, LTE-Advanced, MIMO, Carrier Aggregation, 5G, Massive MIMO, ns-3
\end{abstract}

\section{INTRODUCTION}

Nowadays mobile communications are used by most of the world population. According to [1] at the end of 2016 there were 7.5 billion mobile subscriptions that are expected to reach 8.9 billion by 2022 . LTE will be the dominant technology even after the upcoming of $5 \mathrm{G}$ at the beginning of 2020: in fact, LTE devices are predicted to be 4.6 billion by 2022 , more than half of the whole mobile subscriptions.

This increase of data connections demand, as well as the diffusion of multimedia services, calls for higher data rates than those achievable with LTE. New commercial LTE-A devices are equipped with capabilities providing greater spectral efficiency on a wider spectrum:

- DL 256 Quadrature Amplitude Modulation (256QAM);

- 4x4 Multiple Input Multiple Output;

- 3, 4 or more Component Carrier Aggregation over licensed bands.

The diffusion of LTE-A devices is growing throughout the world and Operators are upgrading mobile networks with configurations able to support 3GPP Rel-12 downlink Category 16, for data rates up to 1 Gbps [2]. Typically, this target data rate is not fully achievable so far because of Operators spectrum fragmentation and commercial devices limitation due to RF components (mainly transceivers). In fact, the maximum number of downlink antenna ports managed by current commercial devices is typically 8 . This number can be exploited in different ways, either using MIMO $4 \mathrm{x} 4$ on two aggregated bands, or using MIMO $4 \times 4$ on one band and MIMO $2 \times 2$ on two bands. The first configuration is theoretically more performing and the maximum throughput is about $800 \mathrm{Mbps}$. When this constraint will be overcome, devices will support MIMO $8 \times 8$ and above to guarantee the target peak data rate for next generation technologies [3].

Two possible approaches open up. The first based on bandwidth broadening and the second on the improvement of the spectral efficiency. At the moment, mobile Operators are trying to figure out which approach or combination of approaches is the best in terms of performance and costs.

The aim of this paper is to study different network configurations in order to evaluate the overall system performance in each case and provide suggestions on the deployment choices to be made. This study was carried on with a network simulator and the results were compared with real measures taken in collaboration with TIM (Telecom Italia) on new commercial devices.

This paper is structured as follows: in Section II we will present the case study and the principal features of the technology. In Section III we describe the method of our analysis and the implementation of the scenario within a network simulator. In Section IV we compare the results of the simulations with measures made on commercial devices over real network. Conclusions form Section V.

\section{REFERENCE SCENARIO}

We consider five celluar sites in a real neighborhood to represent a dense urban scenario. Each site is characterized by three sectors. In this network, the average cell radius is $550 \mathrm{~m}$, leading to an overall coverage of about $4.5 \mathrm{~km}^{2}$.

Our study considers the use of antennas transmitting with a Radio Base Station (RBS) output power of $43 \mathrm{dBm}$ for each radio branch. These antennas are multi-array and can work at all the frequencies needed for carrier aggregation over four bands: 3GPP Band 20 (800 MHz), 3GPP Band 32 (1500 


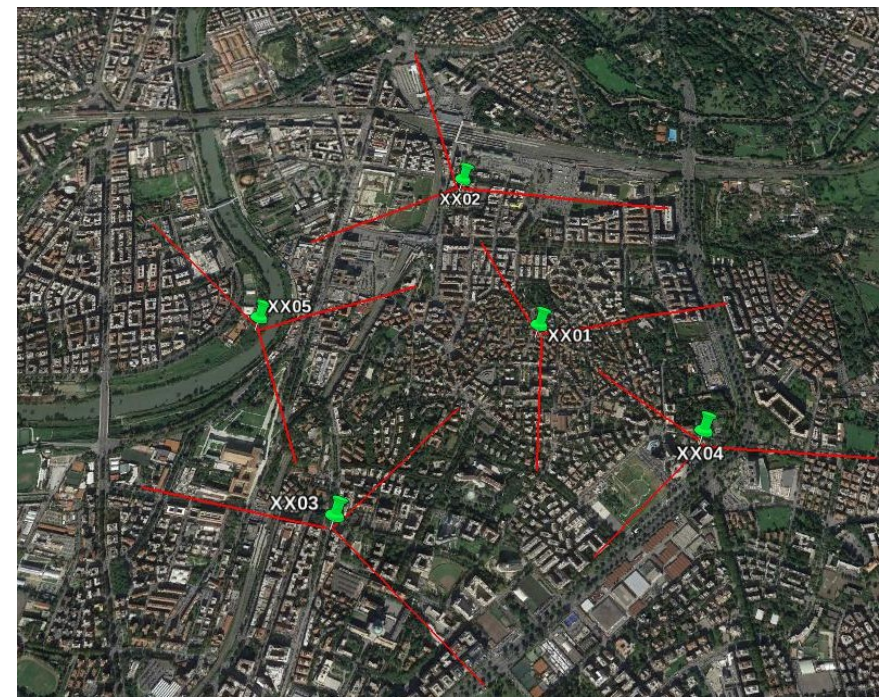

Fig. 1. Scenario

MHz), 3GPP Band 3 (1800 MHz) and 3GPP Band 7 (2600 $\mathrm{MHz}$ ). These antennas can also support both MIMO $2 \times 2$ and MIMO $4 \times 4$. Given the dense urban scenario, we expect around 1000 users per site. Nowadays, video accounts for $50 \%$ of mobile data traffic while in 2022 the percentage will rise up to $75 \%$ [1]. For this reason, we focused our simulations on a high-quality video streaming, that requires a minimum user throughput of $20 \mathrm{Mbps}$.

Among the features described in Section I, we mainly focused the analysis on MIMO and Carrier Aggregation and we examined the advantages and the disadvantages that each feature entails. Here, we give a brief description of both:

a) Carrier Aggregation: CA allows to increase the peak data rate by concatenating several frequency channels in order to transmit on a wider bandwidth, up to $100 \mathrm{MHz}$. This technique has been recently extended to unlicensed spectrum leading to the birth of LTE Licensed Assisted Access (LAA) that can aggregate up to $60 \mathrm{MHz}$ in the $5 \mathrm{GHz}$ spectrum [4].

b) MIMO 4x4: LTE-A Release 10 extended the transmission layers up to 8 in order to guarantee higher data rates. In the future, 5G will use Massive MIMO technologies that will extend the number of simultaneous transmit and received streams [5]. Nowadays, it is very difficult to implement such a complex radio interface on a device and last commercial devices provide only for MIMO $4 \mathrm{x} 4$. It is important to highlight that this techniques is not very robust with respect to noise and requires high SINRs. Note that, apart from the number of antennas, novelties have been introduced with active antennas based on the digital beamforming: it allows to focalize the signal between UE and eNB so that the useful signal increases with respect to the interference.

The broadening of the usable spectrum should lead to significant improvement in terms of performance [6], but mobile network Operators must deal with a limited disposability of bandwidth. Therefore, they encourage to move towards the deployment of massive MIMO but this implies higher manufacturing and implementation costs. Moreover, user equipment side, Operators are still encountering the limit of downlink Antenna Port due to RF components. Since MIMO (both $4 \times 4$ and $8 \times 8$ ) is highly affected by noise, it is not always available to user equipment, especially in a dense urban scenario where reflection and refraction phenomena are frequent, leading to performance departing away from theoretical limits as highlighted in [7].

\section{AnAlysis AND Simulations}

In our analysis, we used $n s-3$, a discrete-event network simulator designed as a set of libraries written in C++ [8]. $n s-3$ is organized in modules (i.e. LTE, Internet) each supplying a single functionality or layer. This tool allows to set the mobility of each UE, so that we could simulate a heterogeneous environment, with some equipment at a fixed position and others moving at a maximum speed of $60 \mathrm{~km} / \mathrm{h}$, which is very likely the maximum achievable speed in a dense urban environment. The radio environment is that described in [9]: this recommendation provides guidance on outdoor short range transmissions (less than $1 \mathrm{~km}$ ) for both line-ofsights (LoS) and non-line-of-sight (NLoS) environments. In our scenario we consider the latter: it takes into account urban canyons, characterized by buildings of several floors each that can significantly contribute to long path delays and a large number of vehicles that may act as reflectors adding Doppler shift to the waves.

We simulated the deployment of DL Category 16 devices that can reach up to 800 Mbps (with DL 256QAM) according to the configuration used between those descibed in Section I.

The simulator provides an output interface to read each transmission parameter: transmitted and received packets and bytes, as well as the throughput associated to a single data flow, then to a single user.

Hence, we evaluated the overall system performances and the effectiveness of Carrier Aggregation and MIMO $4 \times 4$. For the latter we evaluated the percentage of transmission in which it was activated. We set the devices to achieve $20 \mathrm{Mbps}$ as target data rate. In this way we can also evaluate the system at its saturation.

The simulator is continuously renewing and enhancing its features but it doesn't provide the whole LTE-A functionalities. To introduce the DL 256QAM we modified the library that oversees the Adaptive Modulation and Coding: we updated the tables referred to spectral efficiency, CQI, MCS and TBS to those described in [10]. The table that links spectral efficiency to CQI was originally proposed by Qualcomm in [11], but there aren't proposals made so far, then we introduced ours, following the previous one's construction.

In $n s-3$, MIMO is not implemented as the use of multiple antennas in transmission and reception. The model is obtained considering the gain that MIMO schemes bring in the system from a statistical point of view. This solution is based on [12] and only provides for MIMO $2 \times 2$.

Then, we propose another technique to implement MIMO, either with 2 or 4 layers. As 3GPP shows in [10], there 
are precise translation tables to be considered when switching from a single layer to multiple ones. In particular, we focused our attention on tables 7.1.7.2.2-1 and 7.1.7.2.5-1. The former refers to the translation from one layer to two layers while the latter refers to the translation to four layers. From here, it is evident that the usage of MIMO $2 \times 2$ leads to an average doubling of the TBS. Equally, the TBS is quadrupled in presence of MIMO $4 \times 4$. The observation of the relationship between the transport block size and the number of layer led us to implement MIMO schemes as the multiplication of the TBS. We adapted the function GetTbSizeFromMcs so that it returns the TBS doubled (or quadrupled) depending on the MIMO scheme.

Though, since MIMO 4x4 feels the effect of noise more than modulation, it can be activated only with a very good channel quality $(\mathrm{CQI}>14)$. Then we allowed the activation only for MCS higher than 24. In case of a lower channel quality, it is permitted to transmit with a lower rank and, thereby, three layers, in order to guarantee however a faster transmission. This can be done for MCS greater than 21. In the code this consideration is made with an if clause in LteAmc library.

Our proposal to implement Carrier Aggregation is simple: we locate multiple devices or eNBs in the same place and, if necessary, we make them move jointly. This follows the reality: in fact, a device has an antenna for each frequency used to received and/or transmit. One problem would be the unawareness when scheduling resources. Actually, there are two kinds of scheduling procedure: Single-Carrier Scheduler and Cross-Carrier Scheduler [13]. The former is the one used nowadays in real networks and doesn't imply each frequency receiver to be aware one another.

When simulating, we dealt with an implementation problem with the UDP traffic: in fact, within the simualtor, each flow saturates when the data flow, eNB side, is higher than 75 Mbps. We overcame this problem with our implementation of CA: infact, since we use four antennas per sector, we have 12 co-located antennas per site each of which can guarantee at maximum 75 Mbps. Hence, the whole site can provide a 900 Mbps data rate, which is close to the real network bottleneck at $1 \mathrm{Gbps}$.

To design the scenario within the simulator we set the positions of the antennas and the directions of the respective beams to maximize the coverage. The simulator includes a tool to analyze the SINR levels over the considered area, the Radio Environment Map Helper. It is impossible, though, to obtain a general map simultaneously including the contributions of each frequency: in fact, the helper works only at a given frequency for each simulation.

In Fig. 2 we provide the SINR map at $800 \mathrm{MHz}$. Of course, the higher the frequency, the worse is the coverage.

We also made measures on DL Category 16 commercial devices to evaluate both the data rates and the performances of LTE-A features. Unlike the simulations, real measures were made only with the $2 \mathrm{CA}$ configuration with MIMO $4 \times 4$. In fact, the combination of the four LTE carriers available was introduced in the 3GPP Standard in July 2017 within [14]

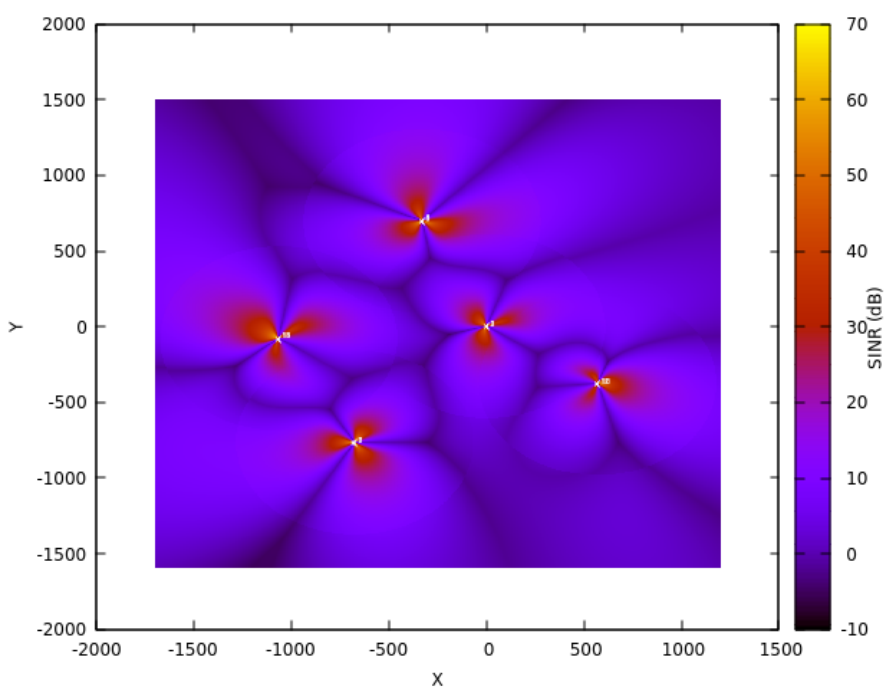

Fig. 2. Coverage at $800 \mathrm{MHz}$

and there are very few commercial devices able to do it yet.

\section{RESUlTS}

With the simulations we analyzed the behavior of the overall network. Tools like $n s-3$ are fundamental for studies like this one, since it is impossible to make such analysis for a real scenario.

First of all, we provide the results of the simulations with the 4-CA configuration that leads to a usage of $65 \mathrm{MHz}$ on the four bands 800, 1500, 1800 and $2600 \mathrm{MHz}$. MIMO 4x4 cannot be used since the limitation to 8 Antenna Port due of RF component on the transceivers.

To evaluate the peak data rate for this configuration we need to consider that over $20 \mathrm{MHz}$, with DL 64QAM and SISO transmission, it is about 75.7 Mbps. Moreover, we need to add the contributions due to MIMO 2x2 and 256QAM. Finally, we have:

$$
\left[\left(75.7 \cdot \frac{65}{20}\right) \cdot 2\right] \cdot \frac{4}{3} \approx 650 \mathrm{Mbps}
$$

The overall system throughput, evaluated as the sum of each UE data rate, is 790 Mbps. From the statistics we got that all the cells and frequencies were used.

The second simulation was focused on the aggregation of only two carriers with MIMO $4 \times 4$ on both. The best configuration is the one that aggregates $40 \mathrm{MHz}$ from 1500 and $1800 \mathrm{MHz}$.

In this way the peak data rate for a single user is

$$
\left[\left(75.7 \cdot \frac{40}{20}\right) \cdot 4\right] \cdot \frac{4}{3} \approx 800 \mathrm{Mbps}
$$

This simulation was mainly focused on evaluating the robustness of MIMO $4 \times 4$ with respect to SINR. To do that, we read through the MAC statistics to evaluate the efficiency as the ratio between the Transport Blocks where MIMO $4 \times 4$ was activated and the whole TBs. 
Since the environment we are simulating is characterised by many users transmitting simultaneously and many multipaths and interferences, it is very hard to achieve a high channel quality and MIMO 4x4 was activated only for the $27 \%$ of Transport Blocks. As comparison, note that 256QAM is activated in the $75 \%$ of the TBs. Hence, the overall system throughput is $630 \mathrm{Mbps}$, lower than the one evaluated in the first configuration, even if this one is theoretically more performing.

To achieve higher SINR levels, it is possible to increase the number of cells. Note that it could lead to a greater intercells interference and to a worsening of data rate. Then, it is important to desing the deployment properly. In fact, it is necessary to reduce the coverage of each cell and to direct the beams in order not to interfere one another. Since it might lead to very high performances, $5 \mathrm{G}$ will provide for the deployment of the so called small cells. In our work, we added a further site to the scenario depicted in Fig. 2 to increase the SINR level. Effectively, MIMO performance was enhanced: it grew to $33 \%$ and the system throughput was $670 \mathrm{Mbps}$.

TABLE I

SiMULATIONS RESULTS

\begin{tabular}{|c|c|c|}
\hline CONFIGURATION & $\begin{array}{l}\text { SYSTEM } \\
\text { THROUGHPUT }\end{array}$ & $\begin{array}{l}\text { SPECTRAL } \\
\text { EFFICIENCY }\end{array}$ \\
\hline $\begin{array}{l}4 \text { CA MIMO } 2 \times 2 \\
\text { 256QAM }\end{array}$ & $790 \mathrm{Mbps}$ & $12.15 \mathrm{bps} / \mathrm{Hz}$ \\
\hline $\begin{array}{l}2 \text { CA MIMO } \\
\text { 256 QAM4 }\end{array}$ & $\begin{array}{l}630 \text { Mbps (670 Mbps } \\
\text { with six sites) }\end{array}$ & $\begin{array}{l}15.75 \text { bps/Hz (16.75 } \\
\text { bps/Hz with six sites) }\end{array}$ \\
\hline
\end{tabular}

It is interesting to highlight that with this configuration only two bands are used (i.e. 1800 and $2600 \mathrm{MHz}$ ) and the network can manage other devices that aggregate other bands like DL Category 6 devices. Then, the complexive capacity increases a lot since the two configuration are independent one another and the throughput of each configuration can be summed and the system capacity increases.

With real measures we evaluated both the peak performance of the devices and the efficiency of MIMO $4 \times 4$. We measured 15 seconds with a $700 \mathrm{Mbps}$ UDP DL traffic. The real maximum throughput is $600 \mathrm{Mbps}$ because of core network limitations that will be overcome by the end of 2017. The throughput trend is shown in Fig. 3 and the main results are in TABLE II.

TABLE II

MAIN MEASUREMENTS

\begin{tabular}{|c|c|}
\hline PARAMETER & VALUE \\
\hline Theoretical TPUT & 691.5 Mbps \\
\hline Measured Peak TPUT & $596.17 \mathrm{Mbps}$ \\
\hline Measured AVG TPUT & $469.83 \mathrm{Mbps}$ \\
\hline B7 MIMO 4x4 \% & $36.1 \%$ \\
\hline B3 MIMO 4x4 \% & $43.8 \%$ \\
\hline
\end{tabular}

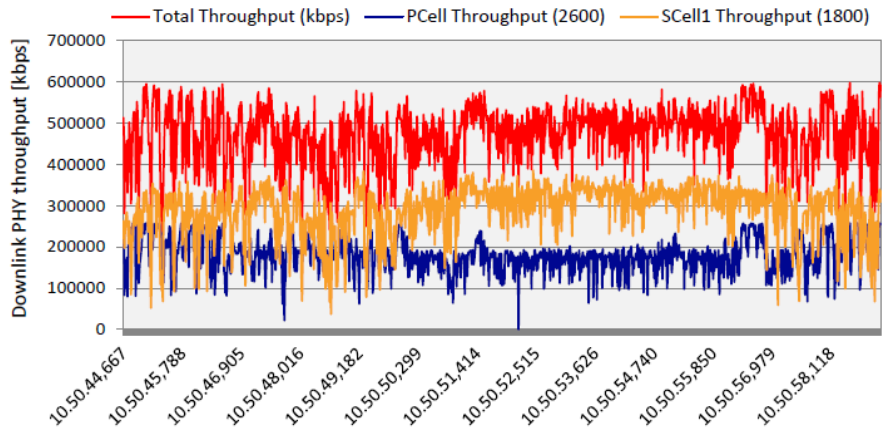

Fig. 3. Throughput trend with B3+B7, 256QAM and MIMO $4 \times 4$

Note that measures were taken in optimal radio conditions. In fact, the average SINR on the four layers is always very high, over both frequencies as we can see in TABLE III.

TABLE III

SINR LEVELS OVER THE 4 LAYERS

\begin{tabular}{|c|c|}
\hline 2600 MHz & AVG \\
\hline SINR RX1 & $29.94 \mathrm{~dB}$ \\
\hline SINR RX2 & $29.27 \mathrm{~dB}$ \\
\hline SINR RX3 & $29.17 \mathrm{~dB}$ \\
\hline SINR RX4 & 29.57 \\
\hline
\end{tabular}

\begin{tabular}{|c|c|}
\hline $1800 \mathrm{MHz}$ & AVG \\
\hline SINR RX1 & $28.54 \mathrm{~dB}$ \\
\hline SINR RX2 & $29.79 \mathrm{~dB}$ \\
\hline SINR RX3 & $29.35 \mathrm{~dB}$ \\
\hline SINR RX4 & 28.76 \\
\hline
\end{tabular}

Then, measures in TABLE II show that MIMOhas a low percentage of activation since it is higly affected by interference, much more than 256QAM that was activated in more than 99\% of transmissions. These results correspond to those obtained within the simulations which highlighted the low efficiency of the 4-layers technique.

Finally, we made a laboratory test to study more deeply MIMO $4 \times 4$. First, we analyzed a system with no correlation between the transmit and receive antennas and we compared the throughput with the two highest modulation schemes. In Fig. 4 we can see that the 4-layers technology effectively introduces the doubling of the downlink throughput. A further increment is given by the adoption of 256QAM as in Fig. 5 .

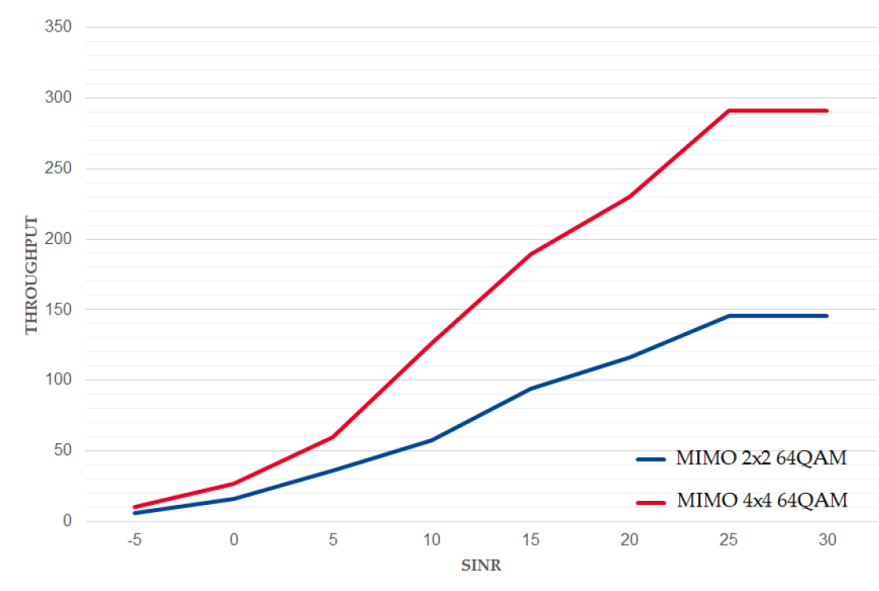

Fig. 4. MIMO $2 \times 2$ vs MIMO $4 \times 4$ 


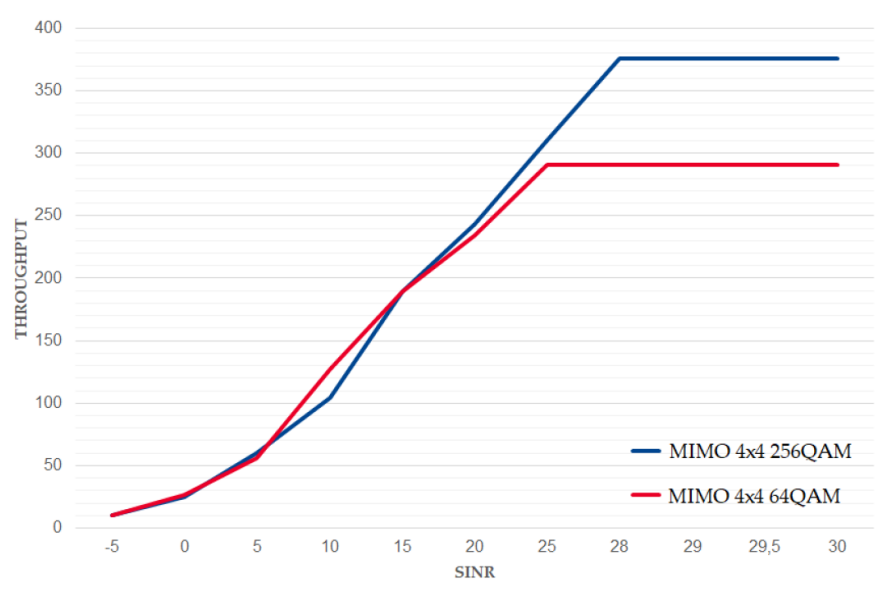

Fig. 5. MIMO 4x4: 64QAM vs 256QAM

Those shown in Fig. 4 and in Fig. 5 are are actually the theoretical behaviors of the multiple-layer transmissions techniques. In fact, if the propagation channels between the antennas can be described as statistically independent and identically distributed, then multiple independent channels can be created by precoding. In practice, this never happens and channels are often correlated and the throughput gain shown in figures above is not achievable.

Then, we made measures in condition of low and medium/high correlation between the paths to reproduce a real radio environment. In fact, in dense urban scenarios like the one we are describing, there are usually many interferences and multipaths. Then, the usage of MIMO $4 \times 4$ might increase reflection and refraction phenomena so that there is not the throughput boost expected. As shown in Fig. 5 theoretical limit with MIMO 4x4 and 256QAM is around $400 \mathrm{Mbps}$ on $20 \mathrm{MHz}$. In Fig. 6, we can see that this value cannot be reached at all, rather it is very far from being achieved, in particular with medium/high correlation.

Even these measures showed the weakness of MIMO 4x4 in real scenarios with multipaths and low signal levels, as it was

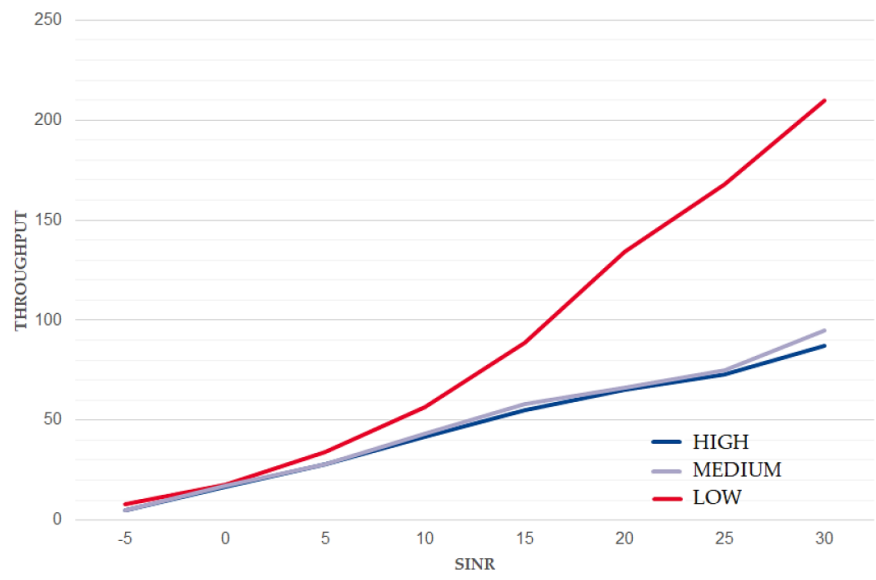

Fig. 6. Comparison among three correlation levels already highlighted by the simulations and general measures. However, in case of optimal channel quality, this technology can give a huge contribution to throughput.

\section{Conclusions}

In this paper we presented an evaluation of the performance of MIMO 4x4 and Carrier Aggregation in LTE-A in diverse scenarios highligthing the limitations and the opportunities that mobile network Operators are dealing with. We also showed the system implementation within a network simulator and the changes made to update it to the last LTE-A Releases. We studied two main configurations in order to evaluate the effectiveness of the LTE-A capabilities and compared the results with real measures to figure out the deployment strategies that mobile Operators should implement. In particular, we focused our attention on new devices that have just entered the market or will be commercial by the end of 2017 .

Simulations results were consistent with the theoretical analysis: the 2-band configuration with MIMO $4 \times 4$ on both bands has even better performances than the 4-band configuration with MIMO $2 \times 2$, but it requires an environment with very low noise. This leads to the need of smaller cells, that, if well dimensioned, would guarantee a better coverage and better signal levels but it would entail elevated deploying costs for Operators.

Hence, we suggest deploying MIMO 4x4 - and 8x8 when it will be available - only in those sites where high SINRs are achievable like indoor scenarios or for those applications that require very high data rates and low latency. Moreover, MIMO 4x4 shall be implemented by Operators with a poor spectrum disposability. Massive MIMO will be the dominant technology for $5 \mathrm{G}$ communications that will provide for very dense cells and very high SINR levels.

Finally, it is interesting to underline that the peak spectral efficiency in the $800 \mathrm{Mbps}$ configuration is $20 \mathrm{bps} / \mathrm{Hz}$ which is comparable to $30 \mathrm{bps} / \mathrm{Hz}$ required by the standard for 5G telecommunications [3], although the restriction of the maximum Antenna Port that will be overcome with the next generation technologies. This to remark that the LTE-A is already near to the best performance that next generation telecommunication networks will fulfill.

Future work will focus on simulating miscellaneous scenarios with diverse user equipment Categories and on comparing the results of the simulations involving the latest technologies with some real measures that are not available yet. Simulator side, the limit on UDP traffic shall be overcome in order to completely fulfil the data rate requirements of new devices. Moreover, it will be necessary to implement MIMO 4x4 (and above) within it.

\section{REFERENCES}

[1] Ericsson, "Ericsson Mobility Report," Ericsson, Tech. Rep., 112016.

[2] 3GPP, "Evolved Universal Terrestrial Radio Access (E-UTRA); User Equipment (UE) radio access capabilities," 3rd Generation Partnership Project (3GPP), TS 36.306, Oct. 2016. [Online]. Available: http://www.3gpp.org/ftp/Specs/html-info/36306.htm 
[3] — "Study on scenarios and requirements for next generation access technologies," 3rd Generation Partnership Project (3GPP), TR 38.913, Oct. 2016. [Online]. Available: http://www.3gpp.org/ftp/Specs/ html-info/38913.htm

[4] - "Feasibility Study on Licensed-Assisted Access to Unlicensed Spectrum," 3rd Generation Partnership Project (3GPP), TR 36.889 , Jul. 2015. [Online]. Available: https://portal.3gpp.org/desktopmodules/ Specifications/SpecificationDetails.aspx? specificationId $=2579$

[5] E. G. Larsson and L. Van der Perre, "Massive MIMO for 5G," IEEE $5 G$ Tech Focus, vol. 1, no. 1, June 2017.

[6] D. Micheli, M. Barazzetta, C. Carlini, R. Diamanti, V. Mariani Primiani, and F. Moglie, "Testing of the Carrier Aggregation Mode for a Live LTE Base Station in Reverberation Chamber," IEEE Transactions on Vehicular Technology, vol. 66, no. 4, pp. 3024-3033, April 2016.

[7] E. Lahetkangas, K. Pajukoski, E. Tiirola, J. Hamalainen, and Z. Zheng, "On the performance of LTE-Advanced MIMO: How to set and reach beyond 4G targets," 18th European Wireless Conference, 2012.

[8] "ns-3." [Online]. Available: http://www.nsnam.org

[9] ITU, "Propagation data and prediction methods for the planning of short-range outdoor radiocommunication systems and radio local area networks in the frequency range $300 \mathrm{MHz}$ to $100 \mathrm{GHz}$,' International Telecommunication Union Radiocommunication Sector (ITU-R), Reccomendation 1411-8, Jul. 2015. [Online]. Available: https://www. itu.int/dms_pubrec/itu-r/rec/p/R-REC-P.1411-8-201507-S!!PDF-E.pdf

[10] 3GPP, "Evolved Universal Terrestrial Radio Access (E-UTRA); Physical layer procedures," 3rd Generation Partnership Project (3GPP), TS 36.213, Jun. 2017. [Online]. Available: http://www.3gpp.org/ftp/ Specs/html-info/36213.htm

[11] 3GPP TSG-RAN WG1, "Conveying MCS and TB size via PDCCH," Apr. 2008. [Online]. Available: http://www.3gpp.org/ftp/tsg_ran/WG1_ RL1/TSGR1_52b/Docs/R1-081483.zip

[12] S. Catreaux, L. J. Greenstein, and V. Erceg, "Some results and insights on the performance gains of MIMO systems," IEEE Journal on Selected Areas in Communications, vol. 21, no. 11, pp. 839 - 847, June 2003.

[13] J. Wannstrom, "Carrier Aggregation explained," Jun. 2013. [Online]. Available: http://www.3gpp.org/technologies/keywords-acronyms/ 101-carrier-aggregation-explained

[14] 3GPP, "Evolved Universal Terrestrial Radio Access (E-UTRA); User Equipment (UE) radio transmission and reception," 3rd Generation Partnership Project (3GPP), TS 36.101, Jul. 2017. [Online]. Available: http://www.3gpp.org/ftp/Specs/html-info/36101.htm 